\title{
Climate change, in the framework of the constructal law
}

\section{Clausse}

Laboratoire de Génie des Procédés pour l'Environnement, l'Energie et la Santé (LGP2ES - EA 21),

CNAM, ICENER, case 2D3P20,

292 rue St. Martin, 75141 Paris Cedex 03, France

and

ESIEE Paris, 2 boulevard Blaise Pascal Cité DESCARTES, BP 99 93162, Noisy le Grand CEDEX, France

E-mail: marc.clausse@cnam.fr

\section{F. Meunier}

Laboratoire de Génie des Procédés pour l'Environnement, l'Energie et la Santé (LGP2ES - EA 21),

CNAM, ICENER, case 2D3P20,

292 rue St. Martin, 75141 Paris Cedex 03, France

E-mail: meunierf@cnam.fr

\section{A.H. Reis*}

Department of Physics and Evora Geophysics Centre, University of Évora, Ramalho, 59, 7000-67 1 Evora, Portugal

E-mail: ahr@uevora.pt

${ }^{*}$ Corresponding author

\section{A. Bejan}

Department of Mechanical Engineering and Materials Science, Duke University, Durham, NC 27708-0300, USA

E-mail: abejan@duke.edu

\begin{abstract}
Here we present a simple and transparent alternative to the complex models of earth's thermal behaviour under time-changing conditions. We show the one-to-one relationship between changes in atmospheric properties and time-dependent changes in temperature and its distribution on earth. The model accounts for convection and radiation, thermal inertia and changes in albedo $(\rho)$ and greenhouse factor $(\gamma)$. The constructal law is used as the principle that governs the evolution of flow configuration on earth. The model showed that for two time-dependent scenarios, $\left(\delta_{\rho}=0.002\right.$; $\left.\delta_{\gamma}=0.011\right)$ and $\left(\delta_{\rho}=0.002 ; \delta_{\gamma}=0.005\right)$ the predicted equatorial and polar temperature increases and the time scales are $\left(\Delta T_{H}=1.16 \mathrm{~K} ; \Delta T_{L}=1.11 \mathrm{~K} ; 104\right.$ years $)$ and $(0.41 \mathrm{~K}$;
\end{abstract}


$0.41 \mathrm{~K} ; 57$ years), respectively. A continuous model of temperature variation was used to predict the thermal response of the Earth's surface to changes bounded by $\delta_{\rho}=\delta_{\gamma}$ and $\delta_{\rho}=-\delta_{\gamma}$ The poleward heat current reaches its maximum in the vicinity of $35^{\circ}$ latitude, accounting for the position of the Ferrel cell between the Hadley and Polar Cells.

Reference to this paper should be made as follows: Clausse, M., Meunier F., Reis, A.H. and Bejan, A. (2012) 'Climate change, in the framework of the constructal law', Int. J. Global Warming, Vol. 4, Nos. 3/4, pp.242-260.

Biographical notes: Marc Clausse received his engineer and MSc degrees form INSA de Lyon (1998) and his PhD from CNAM de Paris in 2003. He is currently Associate Professor at ESIEE Paris and his research focuses on sorption processes for gas separation $\left(\mathrm{CO}_{2}\right.$ capture) and energy conversion, energy efficiency, and more generally the impact of energy use on environment.

Francis Meunier graduated at Ecole Polytechnique Paris. He got his PhD in Solid State Physics (superconductivity) from the Université Paris Sud. He shifted towards solar energy and more generally energy related topics (in particular adsorption heat pumps) in 1977. In 1994, he joined, as a Professor, the Conservatoire National des Arts et Métiers in Paris where his research activity is dedicated to energy and its impact on environment including climate change.

Antonio Heitor Reis graduated with a degree in Physics from the University of Lisbon and received his MSc in Mechanical Engineering from the Technical University of Lisbon (IST) and his PhD in Physics from the University of Évora, Portugal. From 1981 until 1986, he did research on energy at the National Laboratory for Engineering and Technology. He joined the University of Évora in 1986, where he teaches Physics and Engineering courses. He is currently the Director of the Évora Geophysics Centre of the University of Évora. His current research interests comprise energy issues, atmospheric physics, flows in porous media (animate and inanimate) and constructal theory.

Adrian Bejan received all his degrees from MIT BS (1971, Honors Course), MS (1972, Honors Course) and PhD (1975). His research is in Thermodynamics, Applied Physics, and the Constructal Law of Design and Evolution in Nature. $\mathrm{He}$ is ranked by ISI among the 100 most-cited authors in all of Engineering (all fields, all countries, living or deceased). He is the author of 25 books and 550 peerrefereed journal articles. His $\mathrm{h}$ index on ISI is 47 . Professor Bejan was awarded 16 honorary doctorates from universities in 11 countries, for example the Swiss Federal Institute of Technology (ETH Zurich) and the University of Rome I 'La Sapienza'. He received numerous international awards for thermal sciences.

\section{Introduction}

In this paper, we present in simple terms the relationship between changes in average atmospheric properties and time-dependent changes in the distribution of temperature on earth. We focus on the predictable relationship between these two phenomena, not on what may cause changes in the atmosphere. We show that if atmospheric changes are known or predicted, climate response is predictable in concise and transparent terms. 
Two recent articles (Bejan and Reis, 2005; Reis and Bejan, 2006) showed how to use the constructal law to predict the essential features of global climate and atmospheric and oceanic circulation. This was demonstrated in simple and direct terms (with pencil and paper) by combining the constructal law with a simple model of the earth as a heat engine connected to a brake, i.e., an engine with zero delivery of mechanical power to an external user. In accordance with the constructal law, the access of the convective heat current flowing from the warm zone of the earth to the cold zones was maximised. This was achieved by properly sizing the areas of the warm and cold zones, i.e., by generating the configuration (the design) of the flow system and from this were derived the basic climate features, which match observations.

The work described in Bejan and Reis (2005) and Reis and Bejan (2006) was based on steady-state and quasisteady (periodic, diurnal) models. Its success recommends its application to elucidating what is perhaps the biggest issue in geophysics today: climate change, its time scale and how to make its treatment and understanding accessible with pencil and paper, or chalk on the blackboard. In this article, we unveil the relationship between changes in the radiative properties of the atmosphere (albedo, greenhouse factor) and time-dependent changes in the global climate. The key features of this relationship are the time scale and magnitude of the changes.

Before plunging into details, we draw attention to the two significant bodies of literature to which this new work belongs. First, the constructal law has been applied with success to predict patterns in nature in many domains other than global climate, for example, river basins scaling, the distribution of human settlements in geography, animal locomotion (running, flying, swimming, sports evolution), dendritic solidification, turbulent flow structure, human dynamics (e.g., urban design, city traffic), allometric scaling in biology, cracks in shrinking solids, dendritic aggregation of dust particles, heterogeneous multiscale porous media, etc. This literature was reviewed in Bejan (1997, 2000, 2006), Reis (2006) and Bejan and Lorente (2006).

Second, in geophysics there is a significant body of work based on ad hoc invocations of optimality, for example, Maximum Entropy Production (MEP) in Malkus (1954), Lorenz (1955), Paltridge (1975, 1978), Schulman (1977), North (1981), Lin (1982), Swenson (1989) and Lorenz et al. (2001). This work was reviewed in Reis and Bejan (2006) and Whitfield (2006) and is not reviewed again here. It suffices to say that the constructal law is different from MEP because the constructal law is a general statement of physics, about a physics phenomenon that had been overlooked: the time evolution (generation) of flow configuration (design). It is not about the end result (max, min, or optimal), but about the time direction of the evolution phenomenon itself. It applies across the board, from geophysics to biology, engineering and social dynamics.

The constructal law was proposed as a self-standing law of physics in 1996: "For a finitesize flow system to persist in time (to live), its configuration must change in such a way that it provides easier and easier access to the currents that flow through it" (Bejan, 1997). The direction of the 'movie tape' of flow configuration evolution is the law: existing flow configurations are being replaced in time by easier flowing configurations.

\section{Model}

We use the model proposed in Reis and Bejan (2006) (see Fig. 1). The surface temperature is quasi-steady: it is averaged over many daily cycles and annual cycles, but it changes slowly with the changes in the radiative properties of the atmosphere. The surface is not isothermal: 